\title{
Thoracoscopic management of symptomatic esophageal mucocoele after esophageal exclusion
}

\author{
Thapa B, ${ }^{1 *}$ Sapkota $R,{ }^{1}$ Sayami $P^{1}$ \\ ${ }^{1}$ Department of Cardiothoracic Vascular Surgery, Manmohan Cardio-thoracic Vascular and \\ Transplant Center, Institute of Medicine, Tribhuvan University
}

\author{
*Corresponding Author: \\ Dr. Bibhusal Thapa \\ Lecturer , Department of CTVS, \\ Manmohan Cardio-Thoracic Vascular and Transplant \\ center \\ Institute of Medicine, Tribhuvan University \\ Maharajgunj, Kathmandu, Nepal \\ Phone: 9849800099 \\ Email: thapabibhusal@yahoo.com \\ Citation \\ Thapa B, Sapkota R, Sayami P. Thoracoscopic \\ management of symptomatic esophageal mucocoele \\ after esophageal exclusion. Nepal Journal of Medical \\ Sciences 2013;2(2):181-3.
}

\begin{abstract}
Surgical exclusion of the thoracic esophagus is done to avoid mediastinal soiling in esophageal tears when patients present late. Esophageal exclusion can result in the accumulation of secretions and dilatation of the esophageal remnant leading to an esophageal mucocele. Although it is usually asymptomatic, if it increases in size it can produce a variety of compressive symptoms such as coughing, chest pain and respiratory distress. We present a case of symptomatic mucocele after esophageal exclusion treated successfully with thoracoscopic resection. Surgical resection should be considered for symptomatic patients, and thoracoscopy offers minimally invasive and less painful alternative to thoracotomy in these patients.
\end{abstract}

Keywords: Esophageal mucocoele; resection; thoracoscopy

\section{Introduction:}

Surgical exclusion of thoracic esophagus can lead to accumulation of secretions in the closed esophageal segment, with resultant formation of esophageal mucocele. This complication is rarely symptomatic. However, when sufficiently large, the patient may present with coughing, chest and abdominal pain and vomiting. ${ }^{1}$ Life-threatening respiratory distress related to tracheo-bronchial compression is also possible. Infectious complications may occur, resulting in fistulization or septic presentation. ${ }^{2,3}$ Although esophageal exclusion is not an uncommon procedure at our center, symptomatic esophagocoele has been very uncommon. The reported case represents our first successful management of this unusual problem.

\section{Case report:}

A 37year old journalist was referred from the emergency department of the Tribhuvan University Teaching Hospital with a history of epigastric pain and fever of seven days duration. The pain had been preceded by severe retching and vomiting following an alcoholic binge. On examination he was found to have subcutaneous emphysema in the neck. Chest X-Ray showed bilateral pleural effusion. Esophagoscopy confirmed a transmural tear in the lower thoracic esophagus. Esophageal exclusion with a cervical esophagotomy and ligation of the esophago-gastric junction was done along with a feeding jejunostomy was done. This was chosen because the patient was septic at the time of presentation. Six weeks later the patient underwent gastric pull-up. The gastric tube was placed retrosternally. The patient had a smooth post-operative period. He did require two sittings of dilatation of the esophago-gastric anastamosis.

Five months later, the patient represented with complaints of epigastric discomfort and dysphagia. He had lost eight kilograms since last follow up two months ago. An endoscopy revealed a nodular bulging in the first part of the duodenum. A contrast CT scan was done and showed 
a hugely dilated esophageal remnant causing pressure on the adjacent structures. (Figures 1\&2) The patient was taken to theatre. Three port thoracoscopic approach was employed. The mucocoele was first confirmed by aspiration. The esophageal remnant was nearly totally excised leaving only the highest part of the supra-azygous segment. The post-operative period was uneventful and the patient was discharged on the seventh post-operative day.

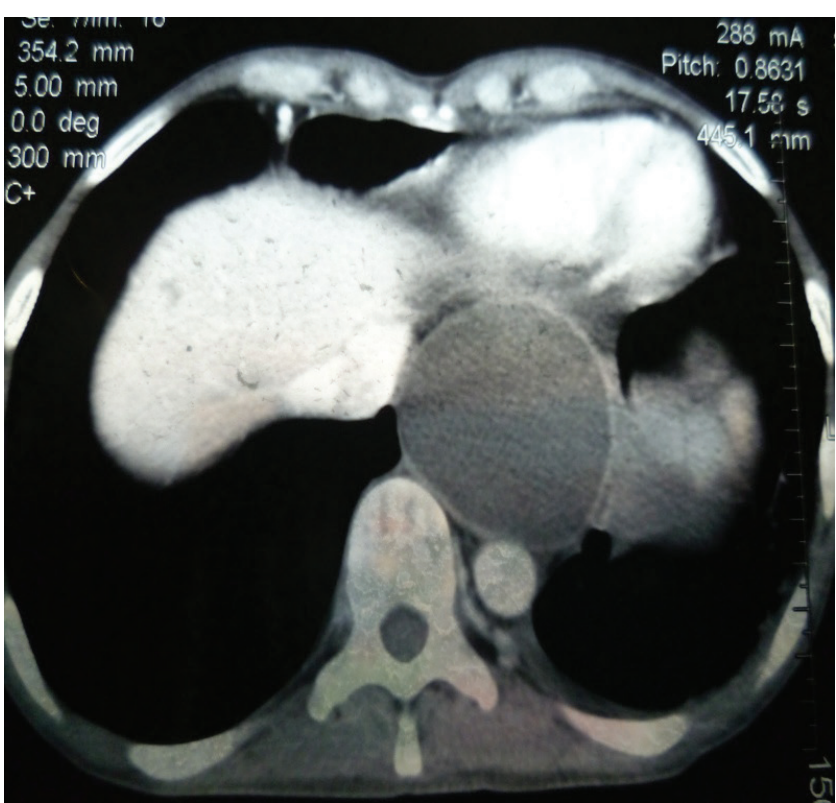

Figure 1: Axial section showing huge esophageal mucocoele

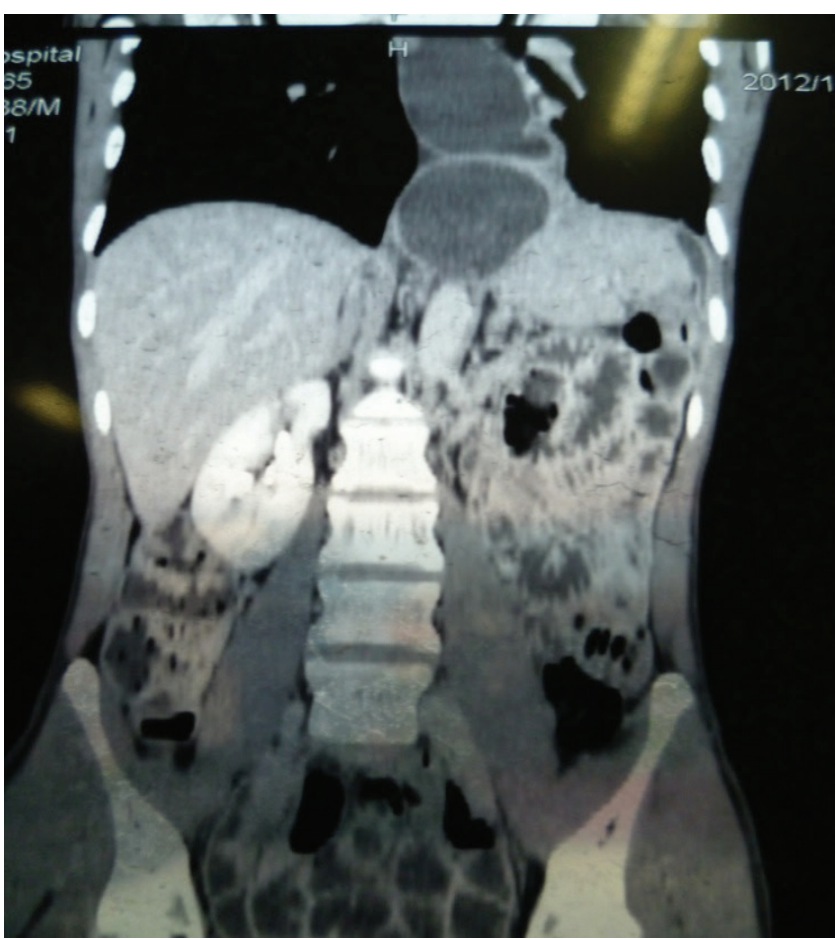

Figure 2: Coronal section showing dilated esophagus.

This case represents the first symptomatic esophageal mucocoele needing surgical treatment encountered at our center. With the application of thoracoscopic surgery, we were able to treat successfully this unusual problem by a minimally invasive technique. This is to the best of our knowledge the first such instance in the country.

\section{Discussion:}

Thoracic esophageal perforation is an emergency with fatality rates approaching 30-50 \% when treatment is delayed. ${ }^{4}$ Emergency surgery in the form of oesophagectomy is not only a way of extirpating the local sepsis in the thoracic cavity, but also of eliminating the dual soilage from the oral cavity and the stomach. ${ }^{5}$ Esophagectomy is thought to be better than conservative surgery in this situstion. ${ }^{5}$ Bipolar exclusion of the esophagus allows prevention of continued mediastinal soiling but is not as extensive and morbid as esophagectomy. This is considered a viable option when primary repair of the esophageal tear is not possible due to late presentation and on-going sepsis. ${ }^{6}$

The formation of a secondary esophageal mucocele is a late sign of incomplete destruction of the esophageal wall. Most esophageal mucocele remain small and asymptomatic. It is possible that the esophageal glands atrophy in response to the increased pressure within the esophageal remnant, makes progressive growth unlikely to occur. On the other hand, as a mucocele enlarges, it can compress or narrow the trachea, causing respiratory symptoms such as coughing, dyspnea and respiratory distress. It can be also related to the development of tracheo-esophageal fistula. ${ }^{1}$ High degree of suspicion is necessary but investigation like Chest X-ray showing a widened mediastinum should lead to a contrast CT scan which confirms the diagnosis.

In our case suspicion aroused by the endoscopy findings were confirmed by the CT scan. Thoracoscopic resection of the esophageal remnant was chosen as the procedure of choice so as to give the patient the benefit of minimal invasion while attaining the therapeutic goal of releasing the pressure caused by the dilated esophagus. Resection of the esophageal remnant was done via thoracotomy in most reported cases. ${ }^{2,5,7}$ We believe that doing it through the thoracoscopic approach help reduce the morbidity and facilitates quick recovery.

Conclusion:This is our first experience with thoracoscopic management of esophageal mucocoele but we can safely conclude that surgery is indicated when the mucocoele becomes symptomatic and thoracoscopy can be considered a viable and less morbid approach. 
Case Report | Thapa B, et al. Thoracoscopy for esophageal mucocoele

\section{Conflict of interest: None}

\section{References:}

1. Haddad R, Lima RT, Boasquevisque $\mathrm{CH}$, et al. Symptomatic mucocele after esophageal exclusion. Interact CardioVasc Thorac Surg 2008;7:742-4.

2. Fong DG, Bueno R, Thompson CC. Esophageal mucocele presenting as a draining fistula after colonic interposition. Gastrointest Endosc 2006;64:290-1.

3. Van Till JWO, Van Sandick JW, Lopes Cardozo M, et al. Symptomatic mucocele of a surgically excluded esophagus. Dis Esophagus 2002;15:96-8.

4. Pate JW, Walker WA, Cole FH Jr, et al. Spontaneous rupture of the esophagus: a 30-year experience. Ann
Thorac Surg 1989; 47:689-92.

5. Okonta KE, Kesieme KB. Is oesophagectomy or conservative treatment for delayed benign oesophageal perforation the better option? Interact CardioVasc Thorac Surg 2012;15:509-5.

6. Khan AZ, Strauss D, Mason RC. Boerhaave's syndrome: diagnosis and surgical management. Surgeon 2007;5:39-42.

7. Fung EW, Gan KD, Lacson A, et al. Esophageal mucoceles causing airway obstruction as a complication of esophageal diversion: two rare pediatric cases and a review of the literature. Int J Pediatr Otorhinolaryngol 2008;72:1563-8. 